\title{
On the Canonical Formalism for a Higher-Curvature Gravity
}

\author{
Yasuo Ezawa ${ }^{1}$, Masahiro Kajihara ${ }^{1}$, Masahiko Kiminami ${ }^{1}$, Jiro Soda ${ }^{2}$ and Tadashi Yano ${ }^{3}$ \\ ${ }^{1}$ Department of Physics, Ehime University \\ Mtsuyama 790 Japan \\ ${ }^{2}$ Department of Fundamental Sciences, FIHS Kyoto University, Kyoto 606 Japan \\ ${ }^{3}$ Department of Electrical and Electronic Engineering, Ehime University \\ Matsuyama, 790 Japan
}

\begin{abstract}
Following to the method of Buchbinder and Lyahovich, we carry out a canonical formalism for a higher-curvature gravity theory in which the Lagrangian density $\mathcal{L}$ is given in terms of a function of the scalar curvature $R$ as $\mathcal{L}=\sqrt{-\operatorname{det} g_{\mu \nu}} f(R)$. The local Hamiltonian is obtained by a canonical transformation which interchanges a pair of the generalized coordinate and their canonical momentum coming from the time derivative of the metric.
\end{abstract}

\section{$\S 1$. Introduction}

The higher curvature gravity (HCG) theory is derived naturally from the superstring theory as its low energy effective theory. HCG is one of the modifications of the Einstein gravity. For macroscopic phenomena, the Einstein gravity works sufficiently well so that the modification is negligible. The modification is, however, expected to play an important role in microscopic, i.e. Planck scale phenomena. In most of the unified theories of fundamental physics, such as superstring, supergravity theory and Kaluza-Klein theories, etc. extra-dimensional spaces are necessary. The extra-dimensional spaces are expected to have contracted to Planck scale during inflation. Then there arises the problem of the stabilization of the extra-dimensional spaces which has been investigated by many authors [1, 2, 3, 4, 5]. In the Einstein gravity, it seems to be difficult to stabilize the extra-dimensional spaces after inflation [6, 7]. So it is natural to ask whether these spaces are stabilized in HCG.

If HCG plays a crucial role in the stabilization, the mechanism would be quantum mechanical. Then we are lead to the canonical formalism of HCG which has been investigated by Boulware 8] and by Buchbinder \& Lyahovich (BL) [9]. The latter authors generalize the formalism of the higher-derivative theory by Ostrogradski to an appropriate form for HCG. They modify the Lagrangian density, (2.1) given below, by using the Lagrange multiplier method which takes into account the definitions of new coordinates and their relations to the time derivatives of the original coordinates. The advantages of the method are that, once the modified Lagrangian density is constructed, all the equations are derived by the variational principle and the consistency conditions, and that it is not necessary that the orders of the highest time derivatives of the coordinates are the same. Thus the procedure is straightforward although tedious. They found that, if the additional term in the modified Lagrangian contains only the square of the scalar curvature, there exist second class con- 
straints. However, they have not obtained the Lagrange multipliers and the final form of the Hamiltonian constraint.

In the formalism of Boulware, the Lagrangian density is restricted to the case in which it contains terms constructed from the Ricci tensor. The generalization to the Lagrangian density given by (2.1) is not straightforward [11, 12].

In this work we will complete the formalism of BL. We reduce the phase space by using the second class constraints. We have only one remaining coordinate arising from the time derivative of the metric 9]. The last step is the canonical transformation which interchanges the new generalized coordinate and its conjugate mementum. This transformation avoids the non-locality when we proceed to the quantum theory. The Hamiltonian thus obtained has more natural dependence on the coordinate and momentum than before the transformation. We will also comment on the relation to the so called equivalence theorem [13, 14] well known in classical HCG.

In section 2, we develope the canonical formalism for the HCG. In section 3, the formalism is applied to the Robertson-Walker spacetime. Section 4 is devoted to the summary and discussion. The problem of stabilization of the extra-dimensional spaces will be investigated in a separate paper.

\section{§2. Canonical Formalism}

In this section we present a canonical formalism of a HCG whose Lagrangian density is given by

$$
\mathcal{L}=\sqrt{-\operatorname{det} g_{\mu \nu}} f(R)
$$

where $f(R)$ is a function of the scalar curvature $R$. In terms of the ADM variables 10 , the three dimensional metric $h_{i j} \equiv g_{i j}$, the lapse function $N$ and the shift vector $N^{i}$, the scalar curvature $R$ is expressed in the following $d+1$ form:

$$
R=2 g^{m n} \mathcal{L}_{n} K_{m n}+\mathbf{K}^{2}-3 K_{m n} K^{m n}+\mathbf{R}-2 N^{-1} \Delta N
$$

Here $K_{k l}$ is the extrinsic curvature

$$
K_{k l} \equiv \frac{1}{2} \mathcal{L}_{n} h_{k l}=\frac{1}{2 N}\left(\partial_{0} h_{k l}-N_{k ; l}-N_{l ; k}\right),
$$

$n^{\mu}$ is the normal to the surface $t=$ constant

$$
n^{\mu}=N^{-1}\left(1,-N^{m}\right)
$$

and $\mathcal{L}_{n}$ is the Lie derivative with respect to the normal $n^{\mu}$

$$
\mathcal{L}_{n} K_{k l}=N^{-1}\left(\partial_{0} K_{k l}-N^{j} K_{k l ; j}-N_{; k}^{j} K_{j l}-N_{; l}^{j} K_{k j}\right)
$$

In the above (and hereafter), a bold face letter denotes the trace of a 2 nd rank tensor, e.g. $h_{k l} R^{k l} \equiv \mathbf{R}$.

Now following BL, we introduce new generalized coordinates

$$
Q_{i j} \equiv K_{i j}
$$


and "velocity" variables

$$
v_{i j} \equiv \partial_{0} Q_{i j}, \quad v^{i} \equiv \partial_{0} N^{i}, \quad \text { and } \quad v \equiv \partial_{0} N
$$

Then there are relations between the new coordinates and the time derivatives of the original coordinates

$$
\partial_{0} h_{i j}=2 N Q_{i j}+N_{i ; j}+N_{j ; i}
$$

In terms of the new variables, Lagrangian density (denoted as $\mathcal{L}_{Q}$ ) is expressed as

$$
\mathcal{L}_{Q}=\sqrt{\operatorname{det} h_{i j}} N f\left(R_{Q}\right)
$$

where

$$
R_{Q}=2 N^{-1} h^{k l} v_{k l}-U_{Q}-2 N^{-1} \Delta N-2 N^{-1}\left(N^{k} \mathbf{Q}_{; k}+2 N^{k ; l} Q_{k l}\right)
$$

and

$$
U_{Q}=3 Q_{m n} Q^{m n}-\mathbf{Q}^{2}-\mathbf{R} .
$$

Using the method of Lagrange multiplier, modified Lagrangian density $\mathcal{L}^{*}$, which takes into account the relations (2.8) and the definitions (2.6) and (2.7), is given as follows:

$$
\begin{aligned}
\mathcal{L}^{*}= & \mathcal{L}_{Q}+p^{k l}\left\{\partial_{0} h_{k l}-\left(2 N Q_{k l}+N_{k ; l}+N_{l ; k}\right)\right\} \\
& +P^{k l}\left(\partial_{0} Q_{k l}-v_{k l}\right)+P_{k}\left(\partial_{0} N^{k}-v^{k}\right)+P\left(\partial_{0} N-v\right) .
\end{aligned}
$$

The Hamiltonian density $\mathcal{H}^{*}$ corresponding to $\mathcal{L}^{*}$ is given as usual

$$
\begin{aligned}
\mathcal{H}^{*} & \equiv P^{k l} \partial_{0} Q_{k l}+P_{k} \partial_{0} N^{k}+P \partial_{0} N+p^{k l} \partial_{0} h_{k l}-\mathcal{L}^{*} \\
& =p^{k l}\left(2 N Q_{k l}+N_{k ; l}+N_{l ; k}\right)+P^{k l} v_{k l}+P_{k} v^{k}+P v-\mathcal{L}_{Q} .
\end{aligned}
$$

The action $S$ to be used in the variational principle is given by

$$
S=\int d^{d+1} x \mathcal{L}^{*}
$$

The variations of the velocitry variables give the "momenta" $P^{k l}, P_{k}$ and $P$ which are expressed as凹

$$
\left\{\begin{array}{l}
P^{k l}=\frac{\partial \mathcal{L}_{Q}}{\partial v_{k l}}=2 \sqrt{h} f^{\prime}\left(R_{Q}\right) h^{k l} \\
P_{k}=\frac{\partial \mathcal{L}_{Q}}{\partial v^{k}}=0 \\
P=\frac{\partial \mathcal{L}_{Q}}{\partial v}=0
\end{array}\right.
$$

where $f^{\prime}=d f / d R$ and $h=\operatorname{det} h_{i j}$. The velocity variables in (2.13) should be replaced by these momenta. Equation (2.15), however, cannot be solved for all velocity variables, i.e.

\footnotetext{
1 In the Ostrogradski's formalism, these equations define the momenta canonically conjugate to the highest time derivatives of the generalized coordinates.
} 
there are primary constraints. Decomposing the second rank tensor (density) into a traceless part (denoted by a tilde) and a trace part

$$
T_{k l}=\tilde{T}_{k l}+\frac{1}{d} h_{k l} \mathbf{T}
$$

we obtain the following expressions for these constraints

$$
\left\{\begin{array}{l}
C^{k l} \equiv \tilde{P}^{k l} \approx 0 \\
C_{k} \equiv P_{k} \approx 0 \\
C \equiv P \approx 0
\end{array}\right.
$$

where $k, l$ run from 1 to $d$ and $\approx 0$ means that the quantity vanishes due to the constraints. The only velocity variable that can be solved from (2.15) is the trace of $v_{k l}$

$$
\mathbf{v}=\frac{N}{2}\left[\psi(\mathbf{P} / 2 d \sqrt{h})+U_{Q}\right]+\Delta N+N^{k} \partial_{k} \mathbf{Q}+2 N^{k ; l} Q_{k l} .
$$

where $\mathbf{P}$, the trace part of $P^{k l}$, is given as

$$
\mathbf{P}=2 d \sqrt{h} f^{\prime}\left(R_{Q}\right) \quad \text { or } \quad R_{Q}=f^{\prime-1}(\mathbf{P} / 2 d \sqrt{h}) \equiv \psi(\mathbf{P} / 2 d \sqrt{h})
$$

Using (2.19), we can express $\mathcal{H}^{*}$ as

$$
\begin{aligned}
\mathcal{H}^{*} & =C^{k l} \tilde{v}_{k l}+C_{k} v^{k}+C v \\
& +N\left[2 p^{k l} Q_{k l}+\frac{1}{2 d} \mathbf{P} \psi(\mathbf{P} / 2 d \sqrt{h})+\frac{1}{2 d} \mathbf{P} U_{Q}-\sqrt{h} f(\psi(\mathbf{P} / 2 d \sqrt{h}))\right]+\frac{1}{d} \mathbf{P} \Delta N \\
& +2 p^{k l} N_{k ; l}+\frac{1}{d} \mathbf{P}\left(N^{k} \partial_{k} \mathbf{Q}+2 N^{k ; l} Q_{k l}\right) .
\end{aligned}
$$

The canonical Hamiltonian density $\mathcal{H}_{0}$ is obtained by putting

$$
\mathcal{H}^{*}=\mathcal{H}_{0}+C^{k l} \tilde{v}_{k l}+C_{k} v^{k}+C v \text {. }
$$

The velocity variables on the right hand side cannot be expressed in terms of the momentum variables and play the role of the Lagrange multipliers. $\mathcal{H}_{0}$ is written in the form

$$
\mathcal{H}_{0}=N \mathcal{H}+N^{k} \mathcal{H}_{k}+\text { divergent terms }
$$

Here

$$
\left\{\begin{array}{l}
\mathcal{H}=2 Q_{k l} p^{k l}+\frac{1}{2 d} \mathbf{P}\left[\psi(\mathbf{P} / 2 d \sqrt{h})+U_{Q}\right]+\frac{1}{d} \Delta \mathbf{P}-\sqrt{h} f(\psi(\mathbf{P} / 2 d \sqrt{h}) \\
\mathcal{H}_{k}=-2 h_{k m} p_{; n}^{m n}+\frac{1}{d}\left[\mathbf{P} \partial_{k} \mathbf{Q}-2\left(\mathbf{P} Q_{k l}\right)^{; l}\right] .
\end{array}\right.
$$

The Poisson bracket(PB) form of the canonical equations of motion are derived from $\mathcal{H}^{*}$ if we treat the pairs of the generalized coordinates and the Lagrange multipliers, $\left(P^{k l}, Q_{k l}\right)$, $\left(p^{k l}, h_{k l}\right),(P, N),\left(P_{k}, N^{k}\right)$, as canonically conjugate pairs. Then it is easily seen that the 
Poisson brackets(PB's) among the primary constraints vanish. Furthermore PB's of $N^{k} \mathcal{H}_{k}$ and the primary constraints also vanish. This means that the time variation of the primary constraints are solely given by $N \mathcal{H}$. Thus the conditions that the primary constraints should be preserved are that the PB's of $N \mathcal{H}$ and the primary constraints should vanish and are expressed by the following set of secondary constraints as

$$
\left\{\begin{array}{l}
\mathcal{C} \equiv \mathcal{H} \approx 0 \\
\mathcal{C}_{k} \equiv \mathcal{H}_{k} \approx 0 \\
\mathcal{C}^{k l} \equiv p^{k l}-\frac{1}{d} \mathbf{p} h^{k l}+\frac{1}{2 d} \mathbf{P}\left(Q^{k l}-\frac{1}{d} h^{k l} \mathbf{Q}\right) \approx 0
\end{array}\right.
$$

The first and the second constraints are interpreted as the "Hamiltonian" constraint and the "momentum" constraint, respectively.

The PB's of the Hamiltonian constraint and the primary constraints also vanishes owing to the secondary constraints. The PB's of the momentum constraint and the primary constraints vanish, whch is desirable if the former one is the generator of the spacial coordinate transformations, which is to be seen. The third one gives the momenta canonically conjugate to $h_{k l}$ which is not given by the equation like (2.13) but was introduced as a Lagrange multiplier. Only non-vanishing PB's among the primary constraints and $\mathcal{C}^{k l}$ is the following:

$$
\left\{C^{k l}(\mathbf{x}), \mathcal{C}^{i j}\left(\mathbf{x}^{\prime}\right)\right\}_{P B}=\frac{1}{2 d} \mathbf{P}\left(h^{k i} h^{l j}-\frac{1}{d} h^{k l} h^{i j}\right) \delta\left(\mathbf{x}-\mathbf{x}^{\prime}\right) .
$$

The constraints $C, C_{k}$ are first class and $C^{k l}, \mathcal{C}^{k l}$ are second class constraints[9]. Thus the velocity variables $\tilde{v}_{k l}$ are determined from the condition that the secondary constraints $\mathcal{C}^{k l} \approx 0$ should be preserved in time:

$$
\begin{aligned}
\tilde{v}_{k l}= & 2 N\left[\left(\mathbf{P}^{-1} \mathbf{p}-\frac{d-1}{2 d} \mathbf{Q}\right) \tilde{Q}_{k l}+Q_{k m} Q_{l}{ }^{m}-\frac{1}{d} h_{k l} Q_{m n} Q^{m n}-\frac{1}{2} \tilde{R}_{k l}\right] \\
& -\mathbf{P}^{-1}\left(\nabla_{k} \nabla_{l}-\frac{1}{d} h_{k l} \Delta\right)(N \mathbf{P}) \\
+ & \tilde{Q}_{k m} N_{; l}^{m}+\tilde{Q}_{l m} N_{; k}^{m}-\left(\tilde{Q}_{k}^{m} N_{l ; m}+\tilde{Q}_{l}^{m} N_{k ; m}\right)+\mathbf{P}^{-1}\left(\mathbf{P} N^{j}\right)_{; j} \tilde{Q}_{k l} \\
+ & 2 \mathbf{P}^{-1}\left(\mathbf{p}+\frac{1}{2 d} \mathbf{P} \mathbf{Q}\right)\left(N_{k ; l}+N_{l ; k}-\frac{2}{d} h_{k l} N_{; j}^{j}\right) .
\end{aligned}
$$

Apart from divergent terms, we have

$$
\begin{aligned}
& C^{k l} \tilde{v}_{k l}=2 N {\left[\left(\mathbf{P}^{-1} \mathbf{p}-\frac{d-1}{2 d} \mathbf{Q}\right) \tilde{P}^{k l} Q_{k l}+\frac{1}{2} \mathbf{P}\left(\mathbf{P}^{-1} P^{k l}\right)_{; k l}+\tilde{P}^{k l} Q_{k m} Q_{l}{ }^{m}-\frac{1}{2} \tilde{P}^{k l} R_{k l}\right.} \\
&+N^{k}\left[2\left\{\left(\tilde{P}_{k}^{m} Q_{m}^{n}\right)_{; n}-\left(\tilde{P}^{m n} Q_{m k}\right)_{; n}\right\}+\mathbf{P}\left(\mathbf{P}^{-1} \tilde{P}^{m n} Q_{m n}\right)_{; k}\right. \\
&+\left.4\left\{\mathbf{P}^{-1}\left(\mathbf{p}+\frac{1}{2 d} \mathbf{P Q}\right) \tilde{P}_{k}^{l}\right\}_{; l}\right]
\end{aligned}
$$


Then we obtain the following form for $\mathcal{H}^{*}$

$$
\mathcal{H}^{*}=C_{k} v^{k}+C v+N \overline{\mathcal{H}}+N^{k} \overline{\mathcal{H}}_{k}+\text { divergent terms }
$$

where

$$
\left\{\begin{aligned}
\overline{\mathcal{H}} & =2 p^{k l} Q_{k l}+\frac{1}{2 d} \mathbf{P} \psi-\sqrt{h} f(\psi)+\mathbf{P}^{-1}\left(\mathbf{p}+\frac{5-d}{2 d} \mathbf{P Q}\right) \tilde{P}^{k l} \tilde{Q}_{k l}+2 \tilde{P}^{k l} \tilde{Q}_{k}^{m} \tilde{Q}_{l m} \\
& +\frac{1}{2 d} \mathbf{P}\left(3 \tilde{Q}_{m n} \tilde{Q}^{m n}+\frac{3-d}{d} \mathbf{Q}^{2}\right)+\mathbf{P}\left(\mathbf{P}^{-1} \tilde{P}^{k l}\right)_{; k l}+\frac{1}{d} \Delta \mathbf{P}-\left(\tilde{P}^{k l} \tilde{R}_{k l}+\frac{1}{2 d} \mathbf{P R}\right) \\
\overline{\mathcal{H}}_{k} & =-2 h_{k m} p_{; n}^{m n}+\frac{1}{d}\left[\mathbf{P} \partial_{k} \mathbf{Q}-2\left(\mathbf{P} Q_{k l}\right)^{l l}\right]+\mathbf{P}\left(\mathbf{P}^{-1} \tilde{P}^{m n} \tilde{Q}_{m n}\right)_{; k} \\
& +2\left[\left(\tilde{P}_{k}^{l}{ }_{k} Q_{l}{ }^{m}\right)_{; m}-\left(\tilde{P}^{m l} \tilde{Q}_{m k}\right)_{; l}\right]+4\left[\mathbf{P}^{-1}\left(\mathbf{p}+\frac{1}{2 d} \mathbf{P Q}\right) \tilde{P}_{k}^{l}\right]_{; l}
\end{aligned}\right.
$$

It appears from eq.(2.29) that it would be more convenient to choose $\tilde{Q}_{i j}$ and $\mathbf{Q}$ as generalized coordinates than $Q_{i j}$. Since we have

$$
P^{i j} \dot{Q}_{i j}=\tilde{P}^{i j} \dot{\tilde{Q}}_{i j}+\frac{1}{d} \mathbf{P} \dot{\mathbf{Q}}+\frac{1}{d}\left(\mathbf{P} Q^{i j}+\tilde{P}^{i j} \mathbf{Q}\right) \dot{h}_{i j},
$$

corresponding canonical momenta are $\tilde{P}^{i j}, \mathbf{P} / d \equiv \hat{\mathbf{P}}$ and $p^{i j}$ which is given by

$$
p^{i j}=p^{i j}+\hat{\mathbf{P}} Q^{i j}+\frac{1}{d} \tilde{P}^{i j} \mathbf{Q}=p^{i j}+\hat{\mathbf{P}}\left(\tilde{Q}^{i j}+\frac{1}{d} h^{i j} \mathbf{Q}\right)+\frac{1}{d} \tilde{P}^{i j} \mathbf{Q} .
$$

It is easily seen that these variables obey the usual Poisson brackets among the canonical pairs of variables. Then $\overline{\mathcal{H}}$ and $\overline{\mathcal{H}}_{k}$ in (2.29) are expressed as

$$
\left\{\begin{aligned}
\overline{\mathcal{H}} & =2 p^{i j}\left(\tilde{Q}_{i j}+\frac{1}{d} h_{i j} \mathbf{Q}\right)+\frac{1}{2} \hat{\mathbf{P}} \psi-\sqrt{h} f(\psi) \\
& -\frac{1}{2} \hat{\mathbf{P}} \tilde{Q}^{i j} \tilde{Q}_{i j}-\frac{d+1}{2 d} \hat{\mathbf{P}} \mathbf{Q}^{2}+\Delta \hat{\mathbf{P}}-\frac{1}{2} \hat{\mathbf{P}} \mathbf{R} \\
& +\frac{1}{d} \hat{\mathbf{P}}^{-1} \mathbf{p}^{\prime} \tilde{P}^{i j} \tilde{Q}_{i j}-\frac{d+1}{2 d} \mathbf{Q} \tilde{P}^{i j} \tilde{Q}_{i j}+2 \tilde{P}^{i j} \tilde{Q}_{i}{ }^{l} \tilde{Q}_{j l}+\hat{\mathbf{P}}\left(\hat{\mathbf{P}}^{-1} \tilde{P}^{i j}\right)_{; i j}-\tilde{P}^{k l} \tilde{R}_{k l} \\
\overline{\mathcal{H}}_{k} & =-2 h_{k m} p_{; n}^{\prime m}+\hat{\mathbf{P}} \mathbf{Q}_{; k} \\
& +\hat{\mathbf{P}}\left(\hat{\mathbf{P}}^{-1} \tilde{P}^{m n} \tilde{Q}_{m n}\right)_{; k}+2\left(\tilde{P}_{k}{ }^{l} \tilde{Q}_{l}{ }^{m}-\tilde{P}^{l m} \tilde{Q}_{k l}\right)_{; m}+\frac{4}{d}\left(\hat{\mathbf{P}}^{-1} \mathbf{p}^{\prime} \tilde{P}_{k}\right)_{; l}^{l}
\end{aligned}\right.
$$

To proceed further, one of the two methods is usually adopted. One is to use only independent variables by solving the constraint equations. The other is to use the Dirac brackets without solving the constraints, which is sometomes more convenient when we cosider symmetry properties. Here we adopt the former method since it is simpler. As to the latter one, we only present the list of the Dirac brackets in the appendix.

Now we reduce the phase space by using the second class constraints, $\tilde{P}^{i j} \approx 0$ and $\tilde{Q}^{i j} \approx 2 \hat{\mathbf{P}}^{-1} \tilde{p}^{i j}$. Remaining independent canonical pairs of variables are $\left(h_{i j}, p^{i j}\right)$ and 
$(\mathbf{Q}, \hat{\mathbf{P}})$, in terms of which $\overline{\mathcal{H}}$ and $\overline{\mathcal{H}}_{k}$ in $(2.31)$ are simplified to

$$
\left\{\begin{aligned}
\overline{\mathcal{H}} & =2 \hat{\mathbf{P}}^{-1}\left(p^{\prime i j} p_{i j}^{\prime}-\frac{1}{d} \mathbf{p}^{\prime 2}\right)+\frac{2}{d} \mathbf{p}^{\prime} \mathbf{Q}+\frac{1}{2} \hat{\mathbf{P}} \psi-\sqrt{h} f(\psi) \\
& -\frac{d+1}{2 d} \hat{\mathbf{P}} \mathbf{Q}^{2}+\Delta \hat{\mathbf{P}}-\frac{1}{2} \hat{\mathbf{P}} \mathbf{R} \\
\overline{\mathcal{H}}_{k} & =-2 h_{k m} p_{; n}^{\prime m n}+\hat{\mathbf{P}} \mathbf{Q}_{; k}
\end{aligned}\right.
$$

In classical theory, (2.32) gives the final form for the Hamiltonian constraint and momentum constraints. However, when passing to the quantum theory, we meet a difficulty with (2.32) in that it contains terms with negative power of $\hat{\mathbf{P}}$. Such terms lead to non-locality in the canonical quantum field theory. Thus we make a canonical transformation which interchanges the momenta with the coordinates

$$
(\mathbf{Q}, \hat{\mathbf{P}}) \longrightarrow\left(\mathbf{Q}^{\prime}, \hat{\mathbf{P}}^{\prime}\right)=(\hat{\mathbf{P}},-\mathbf{Q})
$$

In terms of the new coordinates and momenta, $\psi$ is a function of $\mathbf{Q}^{\prime} / 2 \sqrt{h}$ and $\overline{\mathcal{H}}, \overline{\mathcal{H}}_{k}$ are expressed as

$$
\left\{\begin{aligned}
\overline{\mathcal{H}} & =2 \mathbf{Q}^{-1}\left(p^{\prime i j} p_{i j}^{\prime}-\frac{1}{d} \mathbf{p}^{\prime 2}\right)-\frac{2}{d} \mathbf{p}^{\prime} \hat{\mathbf{P}}+\frac{1}{2} \mathbf{Q} \psi-\sqrt{h} f(\psi) \\
& -\frac{d+1}{2 d} \mathbf{Q} \hat{\mathbf{P}}^{2}+\Delta \mathbf{Q}-\frac{1}{2} \mathbf{Q R} \\
\overline{\mathcal{H}}_{k} & =-2 h_{k m} p_{; n}^{\prime m n}-\mathbf{Q} \hat{\mathbf{P}}_{; k}
\end{aligned}\right.
$$

where we have dropped the primes denoting the new variables for simplicity.

\section{§3. Robertson-Walker spacetime}

In this section we apply the results of the previous section to the Robertson-Walker spacetime whose metric is written as

$$
d s^{2}=-N^{2}(t) d t^{2}+a^{2}(t)\left[\frac{d r^{2}}{1-k r^{2}}+r^{2}\left(d \theta^{2}+\sin ^{2} \theta d \phi^{2}\right)\right] .
$$

The scalar curvature $R$ is given by

$$
R=6 N^{-2}\left[\frac{\ddot{a}}{a}+\left(\frac{\dot{a}}{a}\right)^{2}+\frac{k N^{2}}{a^{2}}-\frac{\dot{N}}{N} \frac{\dot{a}}{a}\right]
$$

The 3-dimensional scalar curvature is $\mathbf{R}=6 k / a^{2}$. The extrinsic curvature $K_{i j}$ is given as

$$
K_{i j}=\frac{\dot{a}}{N a} h_{i j}
$$

where $h_{i j}$ is the 3 -dimensional metric tensor and has a form $a^{2}(t) \times$ (fixed functions of $r, \theta, \phi)$ as can be seen from (3.1). 
The new generalized coordinates $Q_{i j}$ can be taken to be $K_{i j}$ as in the previous section:

$$
Q_{i j} \equiv K_{i j}=\frac{\dot{a}}{N a} h_{i j} .
$$

Obviously there is only one independent component which we take to be the trace part $\mathbf{Q}$ :

$$
\mathbf{Q}=3(\dot{a} / N a) \quad \text { or } \quad Q_{i j}=\frac{1}{3} \mathbf{Q} h_{i j} .
$$

Thus we take $\mathbf{Q}$ to be the new generalized coordinate and the corresponding velocity variables as

$$
\mathbf{v} \equiv \dot{\mathbf{Q}} .
$$

Then the scalar curvature is expressed as

$$
R=2 N^{-1} \mathbf{v}+\frac{4}{3} \mathbf{Q}^{2}+\frac{6 k}{a^{2}} \equiv R_{Q} .
$$

Using this $R_{Q}$, the Lagrangian density is written as

$$
\mathcal{L}_{Q}=\sqrt{-g} f\left(R_{Q}\right)
$$

The modified Lagrangian density, which takes into account the relation (3.5) and the definitions such as (3.6), is given by

$$
\mathcal{L}^{*}=\mathcal{L}_{Q}+\mathbf{P}(\dot{\mathbf{Q}}-\mathbf{v})+P(\dot{N}-v)+p\left(\dot{a}-\frac{1}{3} N a \mathbf{Q}\right) .
$$

Here $\mathbf{P}$ was denoted as $\hat{\mathbf{P}}$ in the previous section and $p$ is related to $p^{\prime i j}$ as $p=2 h_{i j} p^{\prime i j} / a$. It is noted that all the fields are homogeneous. The Hamiltonian density corresponding to this $\mathcal{L}^{*}$ is taken to be

$$
\begin{aligned}
\mathcal{H}^{*} & \equiv \mathbf{P} \dot{\mathbf{Q}}+P \dot{N}+p \dot{a}-\mathcal{L}^{*} \\
& =\mathbf{P} \mathbf{v}+P v+\frac{1}{3} N a p \mathbf{Q}-\mathcal{L}_{Q}
\end{aligned}
$$

Variations with respect to the velocity variables lead to the following equations

$$
\begin{aligned}
& \mathbf{P}=\frac{\delta \mathcal{L}_{Q}}{\delta \mathbf{v}}=2 \sqrt{h} f^{\prime}\left(R_{Q}\right) \\
& P=\frac{\delta \mathcal{L}_{Q}}{\delta v}=0 .
\end{aligned}
$$

From the first equation of (3.11), we have

$$
\mathbf{v}=\frac{1}{2} N\left[\psi(\mathbf{P} / 2 \sqrt{h})-\frac{4}{3} \mathbf{Q}^{2}-6 k / a^{2}\right]
$$

where

$$
\psi(\mathbf{P} / 2 \sqrt{h}) \equiv f^{\prime-1}(\mathbf{P} / 2 \sqrt{h})=R_{Q} .
$$

The second one of (3.11) gives the primary constraint

$$
C \equiv P \approx 0
$$


Then $\mathcal{H}^{*}$ is written as

$$
\mathcal{H}^{*}=C v+\mathbf{P} \mathbf{v}+\frac{1}{3} N a \mathbf{Q} p-\mathcal{L}_{Q}
$$

where

$$
\mathcal{L}_{Q}=N \sqrt{h} f(\psi(\mathbf{P} / 6 \sqrt{h}) .
$$

Using (3.12) we obtain the following expression for $\mathcal{H}^{*}$

$$
\mathcal{H}^{*}=C v+N \mathcal{H}
$$

where

$$
\mathcal{H}=\frac{1}{2} \mathbf{P}\left(\psi-\frac{4}{3} \mathbf{Q}^{2}-6 k / a^{2}\right)+\frac{1}{3} a \mathbf{Q} p-\sqrt{h} f(\psi)
$$

We can treat each pair of variables

$$
(a(t), p(t)), \quad(N(t), P(t)), \quad(\mathbf{Q}(t), \mathbf{P}(t)),
$$

to be canonically conjugate as in the previous section and calculate the Poisson brackets.

Preservation of the primary constraints yields a secondary constraint

$$
0 \approx\left\{C, \mathcal{H}^{*}\right\}_{P . B .}=-\mathcal{H}
$$

This is the so called Hamiltonian constraint. Poisson bracket between $C$ and $\mathcal{H}^{*}$ or $\mathcal{H}$ vanishes, so that we have no additional constraint.

By the canonical transformation which interchanges the coordinate $\mathbf{Q}$ and the momentum $\mathbf{P}$, the final form of $\mathcal{H}$ is obtained

$$
\mathcal{H}=\frac{1}{2} \mathbf{Q}\left(\psi-\frac{4}{3} \mathbf{P}^{2}-6 k / a^{2}\right)-\frac{1}{3} a p \mathbf{P}-\sqrt{h} f(\psi)
$$

where $\psi=\psi(\mathbf{Q} / 2 \sqrt{h})$. Eq.(3.20) is obtained from (2.34) by using the relations $\mathbf{R}=6 k / a^{2}$, $p^{\prime i j}=a p h^{i j} / 2 d$ and putting $d=3$

\section{$\S 4$. Summary and discussion}

Following the method of BL, we presented the canonical formalism of HCG in which the Lagrangian density is given by $\mathcal{L}=\sqrt{-g} f(R)$. Using the second class constraints, we reduced the phase space which is introduced naturally in the BL formalism. At the last step of the procedure, we made a canonical transformation which interchanges the coordinate corresponding to the higher derivatives of the original coordinate and its conjugate momentum. This transformation is necessary to avoid the non-locality when we proceed to the quantum theory. In addition, comparison of the expressions for $\overline{\mathcal{H}},(2.32)$ with (2.34), shows that the dependence of the Hamiltonian on the coordinates and the momenta becomes more natural by this transformation.

The dynamical degrees of freedom of the HCG considered here is just that of the Einstein gravity coupled to one scalar field. The expression of $\overline{\mathcal{H}}$ in $(2.34)$, however, does not appear to describe such a system. We introduce a scalar field $\Phi$ and make a conformal transformation

$$
\Phi \equiv \kappa^{-1} \ln \left(\kappa^{2} \mathbf{Q} / 2 \sqrt{h}\right), \quad \bar{h}_{i j} \equiv\left(\kappa^{2} \mathbf{Q} / 2 \sqrt{h}\right)^{2 / d} h_{i j}
$$


If this is a part of a canonical trasnformation, corresponding canonical momenta $\Pi$ and $\bar{p}^{i j}$ are related to $\hat{\mathbf{P}}$ and $p^{i j}$ as

$$
\left\{\begin{array}{l}
\hat{\mathbf{P}}=\frac{\kappa^{2}}{\sqrt{\bar{h}}}\left[\frac{1}{d} \overline{\mathbf{p}}+\frac{1}{2 \kappa} \Pi\right] \\
p^{i j}=\exp (2 \kappa \Phi / d)\left[\bar{p}^{i j}-\left(\frac{1}{d} \overline{\mathbf{p}}+\frac{1}{2 \kappa} \Pi\right) \bar{h}^{i j}\right] .
\end{array}\right.
$$

Then $\overline{\mathcal{H}}$ takes the following form

$$
\begin{aligned}
\overline{\mathcal{H}} & =\frac{\kappa^{2}}{\sqrt{\bar{h}}}\left[\bar{p}^{i j} \bar{p}_{i j}-\frac{1}{d-1} \overline{\mathbf{p}}^{2}\right]-\kappa^{-2} \overline{\mathbf{R}}+\frac{d-1}{4 d} \frac{1}{\sqrt{\bar{h}}}\left[\Pi-\frac{2 \kappa}{d(d-1)} \overline{\mathbf{p}}\right]^{2} \\
& +\kappa^{-2} \sqrt{\bar{h}}\left[\psi-\frac{1}{2} \exp (-\kappa \Phi) F(\psi)-\{\exp (2 \kappa \Phi / d)-1\} \overline{\mathbf{R}}\right] \\
& +\frac{d+3}{d} \sqrt{\bar{h}} \exp (2 \kappa \Phi / d) \bar{h}^{i j} \partial_{i} \Phi \partial_{j} \Phi
\end{aligned}
$$

where $F \equiv f / 2 \kappa^{2}$. This expression is very similar to the one for the Einstein gravity coupled with a scalar field. The first two terms are just the Hamiltonian of the Einstein gravity. However, the remaining terms are not the one of the scalar field used in the classical equivalence argument. So, at this stage, the equivalence is not clear in the quantum theory, although there might exist a choice of variables which would show the equivalence explicitly.

For the Robertson-Walker spacetime, the second class constraint does not appear since the phase space is reduced from the beginning. This will make the situation simple when we consider the stability of the internal spaces. In this case the second class constraints are restricted to those with respect to the internal variables. Consequently the Dirac brackets are required only for these variables if necessary. Investigation of this problem would require quantum mechanical treatment and will be given in a separate paper.

\section{Appendix. Dirac brackets}

In discussing the symmerty properties of constrained systems, Dirac bracket formalism is sometimes more convenient than the method using only the independent variables. So we here list the Dirac brackets among the canonical variables.

$$
\begin{aligned}
& \left\{h_{i j}(\mathbf{x}), p^{k l}\left(\mathbf{x}^{\prime}\right)\right\}_{D B}=\left(3 \delta_{i}^{k} \delta_{j}^{l}-\frac{2}{d} h_{i j} h^{k l}\right) \delta\left(\mathbf{x}-\mathbf{x}^{\prime}\right) \\
& \left\{h_{i j}(\mathbf{x}), h_{k l}\left(\mathbf{x}^{\prime}\right)\right\}_{D B}=0 \\
& \left\{p^{i j}(\mathbf{x}), p^{k l}\left(\mathbf{x}^{\prime}\right)\right\}_{D B}=0 \\
& \left\{Q^{i j}(\mathbf{x}), P_{k l}\left(\mathbf{x}^{\prime}\right)\right\}_{D B}=\frac{1}{d} h^{i j} h_{k l} \delta\left(\mathbf{x}-\mathbf{x}^{\prime}\right) \\
& \left\{Q^{i j}(\mathbf{x}), Q^{k l}\left(\mathbf{x}^{\prime}\right)\right\}_{D B}=0 \\
& \left\{P_{i j}(\mathbf{x}), P_{k l}\left(\mathbf{x}^{\prime}\right)\right\}_{D B}=0
\end{aligned}
$$




$$
\begin{aligned}
& \left\{h_{i j}(\mathbf{x}), Q^{k l}\left(\mathbf{x}^{\prime}\right)\right\}_{D B}=0 \\
& \left\{h_{i j}(\mathbf{x}), P_{k l}\left(\mathbf{x}^{\prime}\right)\right\}_{D B}=2 d \mathbf{Q}^{-1}\left(h_{i k} h_{j l}-\frac{1}{d} h_{i j} h_{k l}\right) \delta\left(\mathbf{x}-\mathbf{x}^{\prime}\right) \\
& \left\{p^{i j}(\mathbf{x}), Q^{k l}\left(\mathbf{x}^{\prime}\right)\right\}_{D B}=\frac{1}{d} \mathbf{Q}\left(h^{i k} h^{j l}-\frac{1}{d} h^{i j} h^{k l}\right) \delta\left(\mathbf{x}-\mathbf{x}^{\prime}\right) \\
& \left\{p^{i j}(\mathbf{x}), P_{k l}\left(\mathbf{x}^{\prime}\right)\right\}_{D B}=\left[\frac{1}{d}\left(h^{i j} \tilde{P}_{k l}-\tilde{P}^{i j} h_{k l}\right)-\mathbf{Q}^{-1}(2 \mathbf{p}-\mathbf{P Q})\left(\delta_{k}^{i} \delta_{l}^{j}-\frac{1}{d} h^{i j} h_{k l}\right)\right] \delta\left(\mathbf{x}-\mathbf{x}^{\prime}\right) .
\end{aligned}
$$

The Dirac brackets of $h_{i j}, p^{k l}$ and $\mathbf{Q}, \mathbf{P}$ are given as

$$
\begin{aligned}
& \{\mathbf{Q}, \mathbf{P}\}_{D B}=d \delta\left(\mathbf{x}-\mathbf{x}^{\prime}\right) \\
& \left\{h_{i j}, \mathbf{Q}\right\}_{D B}=0 \\
& \left\{h_{i j}, \mathbf{P}\right\}_{D B}=0 \\
& \left\{p^{i j}, \mathbf{Q}\right\}_{D B}=-\frac{1}{d} h^{i j} \mathbf{Q} \delta\left(\mathbf{x}-\mathbf{x}^{\prime}\right) \\
& \left\{p^{i j}, \mathbf{P}\right\}_{D B}=\left(2 \tilde{P}^{i j}-\frac{1}{d} h^{i j} \mathbf{P}\right) \delta\left(\mathbf{x}-\mathbf{x}^{\prime}\right)
\end{aligned}
$$

\section{References}

[1] E. Cremmer, Z.Horvath, L. Palla and J. Scherk, 1977 Nucl. Phys. B127 57.

[2] K. Maeda 1986 Class. Quantum Grav. 3 233, 651.

[3] F. S. Accetta, M. Gleiser, R. Holman and E. W. Kolb 1986 Nucl.Phs. B276 501.

[4] U. Bleyer and A. Zhuk 1995 Gravitation and Cosmology 1106.

[5] V.D.Ivashuchuk and V. N. Melnikov 1995 Gravitation and Cosmology 1133

[6] Y. Ezawa, J. Soda, T. Watanabe and T. Yano, 1996 Nuovo Cimento 111B 355.

[7] Y.Ezawa, F. Kakimoto, J. Soda and T. Yano, 1997 Class. Quantum Grav. 14, 257.

[8] D. G. Boulware, 1984 Quantum Theory of Gravity ed S. M. Christensen(Bristol: Hilger) P 267

[9] I. L. Buchbinder and S. L. Lyahovich, 1987 Class. Quantum Grav. 4, 1487.

[10] R. Arnowitt, S. Deser and C.Misner, 1962 Gravitation: An Introduction to Current Reseach ed. L. Witten(New York: Wiley) P 227

[11] I. L. Buchbinder, I. Yu Karataeva and S. L. Lyakhovich, 1991 Class. Quantum Grav. 8 1113. 
[12] U. Kasper, 1993 Class. Quantum Grav. 10, 869

[13] B.Whitt 1984 Phys. Letters 145B, 176.

[14] K. Maeda 1989 Phys. Rev. D39, 3159. 